\title{
The pathogenesis of airway colonization: lessons learned from the study of bacterial adherence
}

\author{
M.S. Niederman
}

Respiratory infections commonly develop in patients who are already chronically ill, with pneumonia and bronchitis often contributing to further morbidity and mortality. A number of investigators have dedicated their careers to understanding the mechanisms by which chronically and critically ill patients have an increased risk for respiratory infection, and a number of operative mechanisms have been defined. Common to all of these investigations is an awareness that most patients develop invasive pulmonary infection only after preceding colonization of the oropharynx and tracheobronchial tree by the aetiological pathogen. Among hospitalized patients, colonization of the oropharynx by enteric Gramnegative bacteria (EGNB) is common, and occurs at a rate that parallels the degree of systemic illness that is present in a given individual [1]. This event is linked to respiratory infection, because oropharyngeal Gramnegative colonization is often a harbinger of subsequent nosocomial pneumonia, with most pneumonia patients having oropharyngeal colonization before they develop invasive lung infection [2].

One of the mechanisms that ties serious illness to an increased rate of mucosal colonization is bacterial adherence, or the ability of bacteria to bind to receptors on an epithelial cell surface. In studies of oropharyngeal and tracheobronchial colonization, investigators have found that the epithelial cells of colonized patients allow pathogenic Gram-negative bacteria to bind more avidly than epithelial cells of noncolonization do [3-5]. Many of the clinical conditions that are associated with colonization have been shown, in either animal or human studies, to be capable of altering the respiratory mucosal surface in a manner that increases its ability to bind bacteria [3]. Thus, changes in epithelial cell bacterial adherence may be one mechanism by which systemic illness translates into a specific cellular behaviour, leading to an increased risk of airway colonization and pneumonia.

Conceptually, bacterial adherence involves the interaction between adhesins on a bacterial surface and receptors on the epithelial surface. In this framework, colonization results only if certain conditions are met [3]. A putative colonizing organism must have an appropriate adhesin for the mucosal surface presented, and the cell surface must have receptors for the organism. In addition, the host defence system, particularly the mucociliary escalator

Correspondence: M.S. Niederman, Pulmonary and Critical Care Division, 222 Station Plaza N, Suite 400, Mineola, NY, USA 11501. in the tracheobronchial tree, must be impaired sufficiently to allow bacteria to have prolonged contact with the epithelial surface, so that any potential bacterial-mucosal binding interaction will have enough time to occur. This simple paradigm can explain a number of clinical observations about colonization. For example, each mucosal site of the body (lung, skin, urinary tract) has its own unique normal and pathogenic flora. These "tissue tropisms" for certain bacteria may be a manifestation of the fact that different epithelial surfaces contain different types of receptors that can interact with some bacteria, but not others. In the tracheobronchial tree, receptors for a number of pathogenic bacteria (enteric Gram-negative organisms and $H$. influenzae) are present on the epithelial cells and in respiratory mucus, and yet the tracheobronchial tree is normally sterile. In order for colonization to occur, prolonged mucosal contact with bacteria is needed, and, not surprisingly, diseases associated with impaired mucociliary clearance are exactly the conditions that are complicated by chronic airway colonization. Thus, the patient with chronic bronchitis is commonly colonized by $H$. influenzae and $M$. catarrhalis, whilst the patient with cystic fibrosis or bronchiectasis is commonly colonized by $P$. aeruginosa [3].

In studies of bacterial adherence to respiratory cells, experiments are designed to evaluate one of three influences on microbial binding: epithelial cell variables; bacterial variables; and microenvironmental variables [3]. Ideally, each of these influences can be examined by keeping the other two constant, whilst one is manipulated. Thus, keeping bacterial and mixing conditions constant, cells from either different patients or different sites in the respiratory tract can be studied. Using nonmucoid $P$. aeruginosa as a bacterial probe, it has been demonstrated that tracheal cells are able to bind the organism more avidly than buccal cells can (defining a tropism, or preference, of $P$. aeruginosa for the lower respiratory tract), and that the tracheal cells from tracheostomized patients bind more bacteria than cells from normal individuals do [5]. Other variables that have been shown to influence the number and type of epithelial cell receptors for bacteria include the type of systemic illness present, the density of cilia on the tracheobronchial surface, the quantity and structure of cell surface mucins and glycoproteins, and the amount of denuded epithelium exposed as the result of airway injury [3, 6-8]. In addition to species- and strain-specific variability in adhesin composition, other bacterial variables can affect adherence, including: 
the presence or absence of pili or alginate; and the ability of bacteria to produce exoproducts that can interfere with mucociliary clearance or injure the epithelial surface itself. The conditions under which bacteria and cells meet one another can also determine the extent of bacterial adherence that results. Thus, concentrations of proteolytic enzymes and immunoglobulins in sputum, as well as such factors as the $\mathrm{pH}$ of the airway secretions, can all influence bacterial adherence [3].

Many of these concepts are well-demonstrated in the studies reported in the current issue of the Journal by TSANG and co-workers [9] and by RIISE et al. [10]. TSANG and co-workers [9] have presented data concerning the interaction of nonmucoid, piliated strains of $P$. aeruginos $a$ (PA) with organ cultures of human adenoid tissue, an intact epithelial surface that serves as a surrogate for ciliated lower respiratory epithelium. They have shown that bacterial interaction with the mucosa is a dynamic process with the nature of surface receptors changing over time, often as the result of bacterial-induced injury to the organ culture surface. Initially, PA interacted very little with intact mucosa, but after $4 \mathrm{~h}$ the bacteria had injured the normal epithelium and created new surfaces for binding. Once this had occurred, mucosal binding of bacteria increased dramatically. Whilst few bacteria bound to ciliated and non-ciliated epithelial cells, PA bound avidly to surfaces that resulted from bacterial injury to the mucosa. Bacteria bound to ciliated and nonciliated epithelial cells, PA bound well to damaged cells, basement membrane (which became exposed because of injury), to the epithelial surfaces exposed by the loss of tight junctions between cells, and to mucus and cellular debris.

These observations may have pathogenic relevance to airway colonization in a number of clinical conditions. In patients with certain chronic airways diseases (i.e. bronchiectasis), PA could enter the lung and contribute to airway injury, thereby creating favourable conditions for its own persistence. In this way, PA could lead to persistent colonization, which would not only be selfperpetuating but could also contribute to further airway damage. The clinical implication of these mechanisms is that antibacterial agents may be vitally important for the removal of micro-organisms in order to prevent further injury and infection. Similarly, in critically ill patients, the proteolytic enzymes associated with acute lung injury, as well as the mucosal damage and increased mucus production resulting from endotracheal intubation, could contribute to airway colonization by organisms such as PA. With acute illness, airways injury and increased mucus production could create large numbers of tracheobronchial binding sites for bacteria, thereby setting up the lung for subsequent colonization and pneumonia. Once again, if these mechanisms are operative, they suggest a possible benefit for topical tracheobronchial antibiotics in breaking the cycle of injury and infection. The lack of tracheobronchial instillation of antibiotics may, in part, explain why selective digestive decontamination is often unable to eliminate colonization and infection by $P$. aeruginosa, an organism that, for a number of reasons, has a tropism for colonizing the tracheobronchial tree, without first colonizing the oropharynx [11].
Other mechanisms may also contribute to the ability of epithelial cells from critically ill patients to bind Gramnegative bacteria in increased numbers. Nonmucoid PA can bind specifically to tracheal epithelial cells (especially nonciliated cells), and the binding exceeds that seen with mucoid organisms, or with other enteric Gram-negative bacteria (EGNB) [12]. Bacteria can also bind to tracheobronchial mucins, as shown in many studies [7, 8], including the one by TSANG and co-workers [9]. However, this binding may be nonspecific, and in one study investigators were unable to confirm that highly purified mucins from human sputum had specific receptors for PA pili [13]. Mechanical injury to the tracheal surface (as could occur from endotracheal intubation and suctioning), may expose new binding sites for PA [6], and the biofilm inside the endotracheal tube itself may be a surface to which bacteria bind avidly [14]. Once the tracheobronchial surface is injured, the epithelium can repair itself, and in doing so, novel tracheobronchial cell surface carbohydrates may be produced. In one study, these reparative changes allowed for enhanced binding of $P$. aeruginosa to intact organ cultures of ciliated cells [15].

Critical illness and acute lung injury can also alter the composition of cell surface carbohydrates and glycoproteins, thereby creating new and increased numbers of binding sites for Gram-negative bacteria. Both in animal and human studies of critical illness, changes in buccal cell carbohydrates, which may allow for the binding of Gram-negative bacteria with type 1 pili, have been observed [16]. Recently, a mechanism for these observations has been suggested. Patients who are critically ill appear to have exoglycosidase enzymes in their saliva, which can remove peripheral monosaccharides from cell surface oligosaccharides and, thereby, expose new bacterial binding sites [17]. This may occur because of enzymatic removal of buccal cell surface sialic acid and galactose, and such buccal cell surface changes have been observed in studies of critically ill patients [16]. These changes may allow bacteria to gain a foothold in the upper respiratory tract, from which they can reach and then colonize the tracheobronchial tree by binding to injured epithelium and mucus.

Understanding the pathogenesis of colonization and the role of bacterial adherence in patients with chronic bronchitis is more difficult. Some of the same mechanisms may prevail, particularly since $H$. influenzae is an organism capable of producing exoproducts that can lead to both ciliostasis and mucosal injury [18]. In order to fully appreciate the impact of bacterial injury on the mucosa and the ability of this injured surface to bind bacteria, it may be necessary, to study bacterial adherence with an intact epithelial cell layer or with an organ culture. This was not the methodology used by RIISE et al. [10], who studied the binding of $S$. pneumoniae and $H$. influenzae to exfoliated oropharyngeal and tracheobronchial cells of nonsmokers, smokers with nonobstructive bronchitis, and smokers with chronic bronchitis. They observed that $H$. influenzae (but not S. pneumoniae) bound better to tracheobronchial ciliated and goblet cells from patients with nonobstructive bronchitis than to the 
same types of cells from normals or from patients with chronic bronchitis. Although the findings imply a role for adherence in colonization of the airway of smokers, their relationship to the binding of intact epithelial surface is unclear. The lack of an increased binding capacity by tracheobronchial cells from patients with established chronic bronchitis is difficult to understand, unless the pathogenesis of colonization is biphasic. Possibly, smoking leads to epithelial cell changes that increase the ability of the tracheobronchial mucosa to bind bacteria. Once this binding occurs, lung injury results, but the mucosal changes predisposing to adherence regress, or else the mucosa becomes receptive to another type or strain of bacteria.

Further studies with other organisms and both intact and exfoliated mucosal cells may clarify these questions. Tsang and co-workers [19] have used an adenoid organ culture to examine the binding of nontypeable $H$. influen$z a e$, and have observed a dynamic bacterial interaction with the mucosa. When the bacteria were mixed with an intact organ culture, ciliostasis and epithelial injury resulted, and once again these changes could create potential new binding sites for bacteria. Similarly, H. influenzae may be able to synthesize new surface adhesins after binding to an epithelial surface [20]. Thus, both the bacteria and the tracheobronchial epithelium may undergo changes as a result of bacterial interactions with the mucosa, and these changes may lead to persistent colonization.

Studies of the pathogenesis of airway colonization have given us a number of insights into respiratory tract infections. The challenge for the future is to use this information to develop effective prophylactic strategies. The concept of bacterial injury predisposing to persistent colonization and infection has already led to the use of systemic and topical antibiotics in patients with diseases such as bronchiectasis and cystic fibrosis. The optimal antibiotic strategy for chronic bronchitis still needs to be defined, but the use of antibiotics at times of acute exacerbation does appear to be justified. In critically ill patients, studies of the pathogenesis of colonization have suggested a role for prophylactic tracheobronchial antibiotics, but the best clinical strategy for their application has not been carefully characterized. Ultimately, studies of bacterial adherence should lead to novel prophylactic strategies. The use of receptor or adhesin analogues to competitively inhibit binding remains a possibility. The idea of vaccinating patients with adhesin structures to raise antibodies to bacterial surface antigens that lead to colonization has also been considered, but is unlikely to be effective.

Unfortunately, all of the studies of bacterial adherence make it clear that the interaction is a complex one, involving multiple bacterial adhesins and mucosal surface receptors. Therefore, it is unlikely that the binding process can be interrupted by a single intervention, directed at a "universal" adhesin or receptor. Perhaps our awareness of microenvironmental factors that influence bacterial interactions will lead to fruitful prevention strategies, particularly if a widely applicable influence on binding can be defined. Candidates for manipulation include airways $\mathrm{pH}$, sputum mucin content and consistency, and sputum protease activity. Until such strategies are established in clinical practice, investigation into mechanisms of airways colonization will remain important not only because of the insights they have already provided but also because of the obvious clinical benefit that can result from further progress.

\section{References}

1. Johanson WG, Pierce AK, Sanford JP. Changing pharyngeal bacterial flora of hospitalized patients. $N$ Engl J Med 1969; 281: 1137-1140.

2. Johanson WG, Pierce AK, Sanford JP, et al. Nosocomial respiratory infections with Gram-negative bacilli. Ann Intern Med 1972; 77: 701-706.

3. Niederman MS. Gram-negative colonization of the respiratory tract: pathogenesis and clinical consequences. Semin Respir Infect 1990; 5: 173-184.

4. Johanson WG, Higuchi JH, Chaudhuri TR, et al. Bacterial adherence to epithelial cells in bacillary colonization of the respiratory tract. Am Rev Respir Dis 1980; 121: 55-63.

5. Niederman MS, Merrill WW, Ferranti RD, et al. Nutritional status and bacterial binding in the lower respiratory tract in patients with chronic tracheostomy. Ann Intern Med 1984; 100: 795-800.

6. Yamaguchi T, Yamada H. Role of mechanical injury on airway surface in the pathogenesis of Pseudomonas aeruginosa. Am Rev Respir Dis 1991; 144: 1147-1152.

7. Ramphal R, Pyle M. Evidence for mucins and sialic acid as receptors for Pseudomonas aeruginosa in the lower respiratory tract. Infect Immun 1983; 41: 339344.

8. Vishwanath S, Ramphal R. Adherence of Pseudomonas aeruginosa to human tracheobronchial mucin. Infect Immun 1984; 45: 197-202.

9. Tsang KWT, Rutman A, Tanaka E, et al. Interaction of Pseudomonas aeruginosa with human respiratory mucosa in vitro. Eur Respir J 1994; 7: 1746-1753.

10. Riise GC, Larsson S, Andersson BA. Bacterial adhesion to oropharyngeal and bronchial epithelial cells in smokers with chronic bronchitis. Eur Respir J 1994; 7: 1759-1764.

11. Niederman MS, Mantovani R, Schoch P, et al. Patterns and routes of tracheobronchial colonization in mechanically ventilated patients: the role of nutritional status in colonization of the lower airway by Pseudomonas species. Chest 1989; 95: 155-161.

12. Grant MM, Niederman MS, Poehlman MA, Fein AM. Characterization of Pseudomonas aeruginosa adherence to cultured hamster tracheal epithelial cells. Am J Respir Cell Mol Biol 1991; 5: 563-570.

13. Sajjan U, Reisman J, Doig P, Irvin RT, Forstner G, Forstner J. Binding of nonmucoid Pseudomonas aeruginosa to normal human intestinal mucin and respiratory mucin from patients with cystic fibrosis. J Clin Invest 1992; 89: 657-665.

14. Inglis TJ, Millar MR, Jones G, Robinson DA. Tracheal tube biofilm as a source of bacterial colonization of the lung. J Clin Microbiol 1989; 27: 2014-2018.

15. Plotkowski MC, Chevillard M, Pierrot D, et al. Differential adhesion of Pseudomonas aeruginosa to human respiratory epithelial cells in primary culture. J Clin Invest 1991; 87: 2018-2028.

16. Weinmeister KD, Dal Nogare AR. Buccal cells 
carbohydrates are altered during critical illness. Am J Respir Crit Care Med 1994; 150: 131-134.

17. Quinn MO, Miller VE, Dal Nogare AR. Increased salivary exoglycosidase activity during critical illness. Am J Respir Crit Care Med 1994; 150: 179-183.

18. Wilson R, Cole PJ. The effect of bacterial products on ciliary function. Am Rev Respir Dis 1988: 138: S49-S53.
19. Tsang KW, Rutman A, Kanthakumar K, et al. Haemophilus influenzae infection of human respiratory mucosa in low concentrations of antibiotics. Am Rev Respir Dis 1993; 148: 201-207.

20. St. Geme J, Falkow S. Haemophilus influenzae adheres to and enters cultured human epithelial cells. Infect Immun 1990; 58: 4036-4044. 